\title{
Broad spectrum antibiotic use among in-patients at a hospital in Nairobi, Kenya
}

\author{
Kizito M. Mariita ${ }^{1 *}$, Harriet A. Chirima ${ }^{1}$, Carol K. Maina ${ }^{2}$
}

${ }^{1}$ Department of Pharmacy, The Nairobi Hospital, Nairobi, Kenya ${ }^{2}$ Mission for Essential Drugs and Supplies, Nairobi, Kenya

Received: 25 October 2018 Accepted: 29 November 2018

\section{*Correspondence to: \\ Dr. Kizito M. Mariita, \\ Email: mariitakizito@ yahoo.com}

Copyright: (C) the author(s), publisher and licensee Medip Academy. This is an openaccess article distributed under the terms of the Creative Commons Attribution NonCommercial License, which permits unrestricted noncommercial use, distribution, and reproduction in any medium, provided the original work is properly cited.

\begin{abstract}
Background: Antimicrobial resistance is an increasingly serious threat to global public health. While the use of antibiotics is an important contributing factor, there are gaps regarding this in our region. This study aimed to describe the use of nine broad spectrum antibiotics among in-patients of The Nairobi Hospital (TNH) so as to identify opportunities for quality improvement.

Methods: This was a retrospective review of the use of meropenem, ertapenem, imipenem, cefepime, piperacillin, gentamicin, amikacin, vancomycin and teicoplanin among in-patients of TNH from $1^{\text {st }}$ January 2018 to $31^{\text {st }}$ March 2018. Demographic and clinical data of all in-patients who were prescribed these antibiotics during the study period were retrieved from patient files.

Results: There were 301 study participants with a median age (range) of 30years (1day-74years), of whom $161(53.5 \%)$ were male. More than half of the participants were admitted for less than one week and had at least one comorbidity. Meropenem was the most commonly prescribed study antibiotic 123 (40.9\%) followed by amikacin 89 (29.6\%). Respiratory tract infections 125 $(41.5 \%)$ were the predominant indications. Meropenem had the longest mean duration of administration, 6.5days while the aminoglycosides were administered for a relatively shorter duration of about 4.8days. Cultures were done on 187 $(62.1 \%)$ patients though it is only samples of 45 patients that grew an organism, E. coli and Klebsiella sp being the most frequently isolated organisms.

Conclusions: There's a need to strongly intensify implementation of restriction strategies for Meropenem use and introduction of education programs on antimicrobial stewardship targeting all prescribers.
\end{abstract}

Keywords: Aminoglycosides, Broad-spectrum antibiotics, Meropenem, Resistance

\section{INTRODUCTION}

Antimicrobial resistance is a major public health problem especially because antibiotics underpin routine medical practice. Multidrug-resistant organisms are associated with worse therapy outcomes, longer hospital stay and higher treatment costs. In addition, while the resistance of organisms to antibiotics is increasing, very few new antimicrobial drugs are being developed. ${ }^{1}$

In the in-patient set up, an estimated $50 \%$ of antibiotic orders are unnecessary. It is in this setting that the broadest-spectrum antibiotics are being used, and rampantly and where the worse forms of drug resistance are being witnessed. ${ }^{2}$ The use of antibiotics is an important factor contributing to the emergence of antibiotic-resistant bacteria. Carbapenems and other broad-spectrum antimicrobial agents should be limited to patients who have severe or antibiotic resistant infections requiring their use. Whenever it is possible and clinically safe to do so, the narrowest spectrum antibiotic agent available should be used. ${ }^{3}$ Appropriate microbiological tests should be performed prior to commencing antibiotic therapy whenever possible in order to guide ongoing therapy as well as providing important epidemiological surveillance data. $^{2,4}$ 
In Kenya, few hospitals have local antimicrobial guidelines or a restriction policy for antibiotics. In an attempt to control the irrational use of broad-spectrum antimicrobials, TNH introduced measures where these can only be prescribed by doctors, for a limited duration and justification for their use provided. This practice has not been fully adopted and there are many instances when antibiotics considered of last resort are prescribed for infections that could otherwise be treated by first line antibiotics. The use of these antibiotics is at times prolonged probably because tests to allow de-escalation are not done or findings not utilized.

Antibiotic use and antibiotic resistance surveillance systems are essential prerequisites for targeted interventions to cope with the problem of antibiotic resistance. ${ }^{5}$ The purpose of this study therefore was to describe the use of nine selected antibiotics at TNH so as to identify opportunities for quality improvement.

\section{METHODS}

The study was a retrospective review of the use of nine broad spectrum antibiotics among in-patients of TNH from $1^{\text {st }}$ January 2018 to $31^{\text {st }}$ March 2018.

\section{Study area and population}

The study was conducted at TNH, a 350-bed private hospital in Nairobi, Kenya with intensive care units, Surgery, Pediatric, and Medicine departments. An average of about 1500 patients are admitted monthly (TNH records, November 2017). Antibiotics are prescribed on a designated area of a patient's treatment sheet separate from orders of other drugs. Enough space is provided to allow the prescriber to write the indication, dose, frequency, and duration.

Other details that need to be provided here include commencement date and time, if any specimen has been taken to identify the organism and the finding, plus the prescriber's name and signature. All these were to ensure that a prescriber prescribes an antibiotic only when he/she is convinced that a patient needs it.

In addition, antibiotics for prophylaxis are required to be given for not more than 24 hours. carbapenems, glycopeptides, linezolid, capsofungin and colistin have been classified as restricted and these can only be issued for a maximum of five days (to patients in general wards) and of seven days (to critical care units) unless there is justification for prolonged use.

For purposes of this study, meropenem, ertapenem, imipenem, cefepime, piperacillin/tazobactam, gentamicin, amikacin, vancomycin, and teicoplanin use were assessed.

Ethical approval was obtained from TNH ethics and research committee before commencement of the study.
Confidentiality was maintained by using study serial numbers instead of participants' names.

\section{Sample size, inclusion and exclusion criteria}

All in-patients who were prescribed the study antibiotics during the review period were included. Those already receiving the selected antibiotics at the commencement of the study and those that were still admitted at the end of the study period were excluded.

\section{Data collection}

A pre-tested data collection form was used to collect information from patients' files during the study period. The data included patient and clinical information, prescribed antibiotic, the intended use of the antibiotic (prophylaxis, unspecific use, type of infection), duration of treatment and hospitalization and if the antibiotic was deescalated at any point. The end point for each study subject was discharge from hospital or death.

\section{Statistical analysis}

The data was coded and entered into SPSS statistics version 21 for analysis. Continuous variables were summarized as the mean and standard deviation of the mean, or as the median and the ranges. Categorical variables were summarized as frequencies and proportions. Chi-square tests were adopted to determine the association between the variables. P-values of 0.05 or less were considered statistically significant.

\section{RESULTS}

\section{Characteristics of the study participants}

The study participants were mainly male $161(53.5 \%)$ and had a median age (range) of 30years (1day-74years). Those below 18 years were the majority, constituting $41.1 \%$ of the participants. Most of the study participants $162(53.8 \%)$ were admitted for less than a week while more than half had at least one co morbidity (Table 1).

\section{Proportion of patients prescribed the study antibiotics}

Meropenem, given to $123(40.9 \%)$ patients, was the most commonly prescribed study antibiotic followed by Amikacin 89 (29.6\%). Piperacillin as well as the other carbapenems were prescribed to a relatively equal number of patients (Figure 1).

\section{Indication for which study antibiotic prescribed}

Respiratory tract infections were the commonest indication for prescribing the antibiotics. About 60 (20\%) of the prescriptions of the drugs were due to sepsis. Thirty three patients received these antibiotics as prophylaxis against infection while $16(5.3 \%)$ of the patients had no specific indication (Figure 2). 
Table 1: Characteristics of the study participants.

\begin{tabular}{|c|c|c|}
\hline Variable & & n $(\%)$ \\
\hline \multirow{2}{*}{ Gender } & Male & $161(53.5)$ \\
\hline & Female & $140(46.5)$ \\
\hline \multicolumn{2}{|c|}{ Age (median, years [range]) } & $(30[0-108])$ \\
\hline \multirow{4}{*}{$\begin{array}{l}\text { Age group } \\
\text { (years) }\end{array}$} & $0-17$ & $124(41.1)$ \\
\hline & $18-35$ & $45(15.0)$ \\
\hline & $36-59$ & $68(22.6)$ \\
\hline & $\geq 60$ & $64(21.3)$ \\
\hline \multirow{5}{*}{ Department } & Medicine & $116(38.5)$ \\
\hline & Surgery & $41(13.6)$ \\
\hline & Ob/Gyn & $4(1.3)$ \\
\hline & Critical care & $30(10)$ \\
\hline & Pediatrics & $110(36.5)$ \\
\hline \multirow{5}{*}{$\begin{array}{l}\text { Length of admission } \\
\text { (days) }\end{array}$} & $\begin{array}{l}\text { (mean, days } \\
\text { [range]) }\end{array}$ & $10.3(1-52)$ \\
\hline & $1-7$ & $162(53.8)$ \\
\hline & $8-14$ & $73(24.3)$ \\
\hline & $15-21$ & $36(12.0)$ \\
\hline & $\geq 22$ & $30(10.0)$ \\
\hline \multirow{3}{*}{$\begin{array}{l}\text { Number of co } \\
\text { morbidities }\end{array}$} & None & $145(48.2)$ \\
\hline & One & $95(31.6)$ \\
\hline & $\geq$ Two & $61(20.3)$ \\
\hline
\end{tabular}

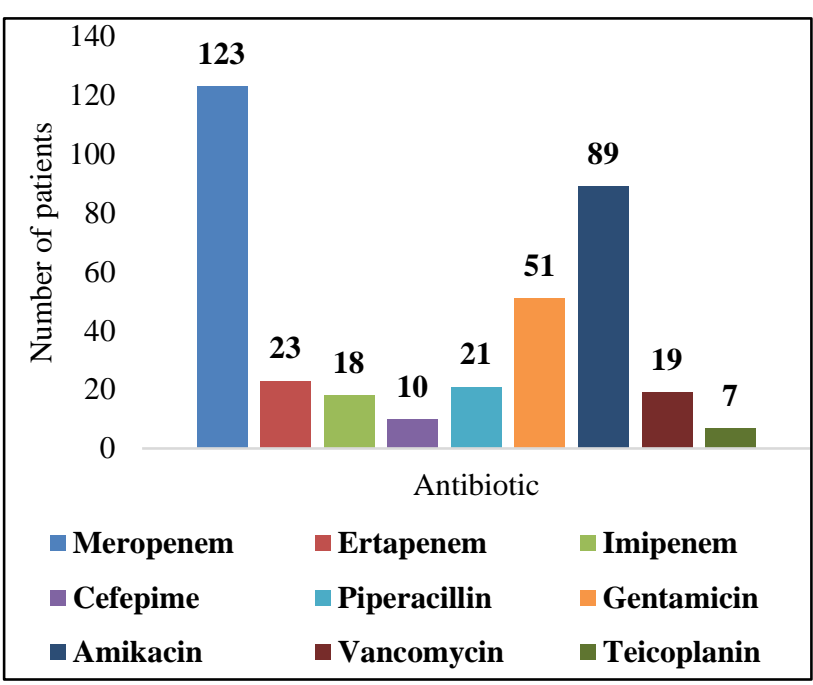

Figure 1: Proportion of use of the study antibiotics.

\section{Duration of use of the study antibiotics}

Meropenem had the longest mean duration of administration ( 6.5 days) followed by cefepime ( 6.4 days) and imipenem (6.1 days). The aminoglycosides were administered for a relatively equal mean duration of about 4 days (Table 2 ).

\section{Identification of causative organisms}

Cultures to try and identify the causative organism were done on $187(62.1 \%)$ patients. However, it is only samples of $45(15.0 \%)$ patients that grew an organism. Escherichia Coli and Klebsiella sp. were the most commonly isolated organisms. Candida albicans was isolated in 8 patient samples. Only 22 patients were switched from broad empirical therapy to a narrow spectrum antibiotic (Table 3).

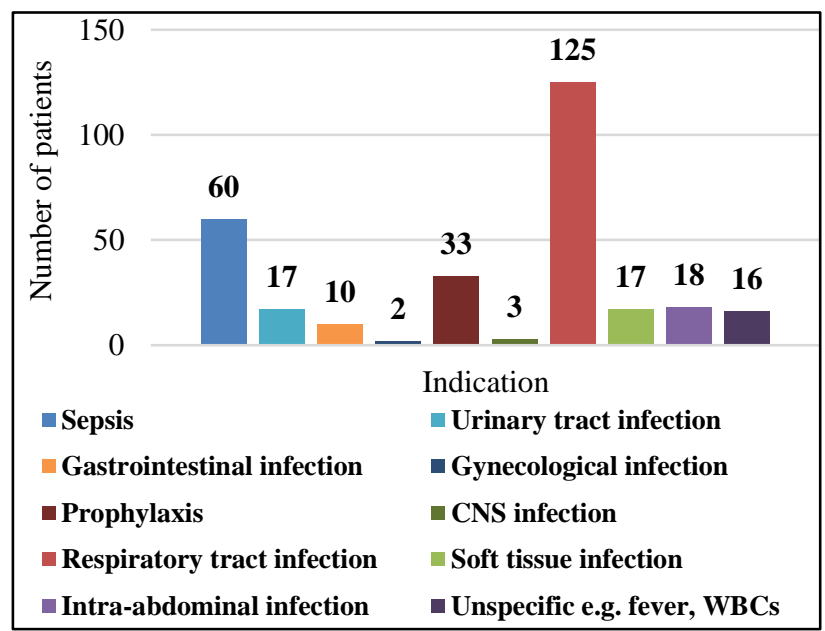

Figure 2: Indication for which study antibiotic prescribed.

Table 2: Duration of use of the study antibiotics.

\begin{tabular}{|ll|}
\hline Antibiotic & $\begin{array}{l}\text { Duration of use } \\
\text { Mean (s.d.) [range] days }\end{array}$ \\
\hline Meropenem & $6.5(3.4)[1-27]$ \\
\hline Ertapenem & $4.4(2.1)[2-10]$ \\
\hline Imipenem & $6.1(2.9)[2-12]$ \\
\hline Cefepime & $6.4(3.9)[1-14]$ \\
\hline Piperacillin/Tazobactam & $5.9(3.1)[1-13]$ \\
\hline Gentamicin & $4.5(2.8)[1-10]$ \\
\hline Amikacin & $4.8(2.7)[1-14]$ \\
\hline Vancomycin & $3.1(3.3)[1-15]$ \\
\hline Teicoplanin & $5.8(4.2)[2-14]$ \\
\hline
\end{tabular}

Table 3: Identification of causative organisms.

\begin{tabular}{|ll|}
\hline Parameter & $\mathbf{N}(\%)$ \\
\hline Cultures done & $187(62.1)$ \\
\hline Organism identified & $45(15.0)$ \\
\hline Staphylococcus aureus & 2 \\
\hline MRSA & 1 \\
\hline Escherichia coli & 11 \\
\hline ESBL E. coli & 2 \\
\hline Klebsiella sp & 8 \\
\hline Pseudomonas aeruginosa & 5 \\
\hline Acinetobacter Baumanii & 1 \\
\hline salmonella typhi & 5 \\
\hline Helicobacter pylori & 1 \\
\hline Serratia marcescens & 1 \\
\hline Fungi & 8 \\
\hline $\begin{array}{l}\text { Patient de-escalated to narrower } \\
\text { spectrum antibiotic }\end{array}$ & $22(7.3)$ \\
\hline MRSA - Methicillin resistant staphylococcus aureus, ESBL - \\
Extended spectrum beta lactamase producing.
\end{tabular}


Table 4: Factors associated with the prescription of meropenem.

\begin{tabular}{|c|c|c|c|c|}
\hline \multirow{2}{*}{\multicolumn{2}{|c|}{ Variable }} & \multicolumn{2}{|c|}{ Meropenem prescribed } & \multirow{2}{*}{$P$ value } \\
\hline & & Yes n (\%) & No $n(\%)$ & \\
\hline \multirow{4}{*}{ Age group (years) } & $0-17$ & $33(11)$ & $91(30.2)$ & \\
\hline & $18-35$ & $20(6.6)$ & $25(8.3)$ & $<0.001$ \\
\hline & $36-59$ & $34(11.3)$ & $34(11.3)$ & \\
\hline & $>60$ & $36(12.0)$ & $28(9.3)$ & \\
\hline \multirow{2}{*}{ Gender } & Male & $70(23.3)$ & $91(30.2)$ & 0.322 \\
\hline & Female & $53(17.6)$ & $87(28.9)$ & \\
\hline \multirow{4}{*}{ Department } & Medicine & $62(20.6)$ & $58(19.3)$ & \\
\hline & Surgery & $13(4.3)$ & $28(9.3)$ & $<0.001$ \\
\hline & Critical care & $22(7.3)$ & $8(2.7)$ & \\
\hline & Pediatrics & $26(8.6)$ & $84(27.9)$ & \\
\hline \multirow{4}{*}{$\begin{array}{l}\text { Length of admission } \\
\text { (days) }\end{array}$} & $1-7$ & $43(14.3)$ & $119(39.5)$ & \\
\hline & $8-14$ & $36(12.0)$ & $37(12.3)$ & $<0.001$ \\
\hline & $15-21$ & $20(6.6)$ & $16(5.3)$ & \\
\hline & $\geq 22$ & $24(8.0)$ & $6(2.0)$ & \\
\hline \multirow{9}{*}{ Indication } & Sepsis & $30(10.0)$ & $30(10.0)$ & \\
\hline & Respiratory infection & $39(13.0)$ & $86(28.6)$ & \\
\hline & Soft tissue infection & $8(2.7)$ & $9(3.0)$ & \\
\hline & Intra-abdominal & $15(5.0)$ & $5(1.6)$ & $<0.001$ \\
\hline & Urinary tract & $10(3.3)$ & $7(2.3)$ & \\
\hline & Gastrointestinal & $7(2.3)$ & $3(1.0)$ & \\
\hline & Prophylaxis & $5(1.7)$ & $28(9.3)$ & \\
\hline & CNS infection & $3(1.0)$ & $0(0.0)$ & \\
\hline & Unspecific & $6(2.0)$ & $10(3.3)$ & \\
\hline \multirow{3}{*}{ Co-morbidities } & None & $45(15.0)$ & $100(33.2)$ & \\
\hline & One & $40(13.3)$ & $55(18.3)$ & $<0.001$ \\
\hline & Two or more & $38(12.6)$ & $23(7.6)$ & \\
\hline
\end{tabular}

\section{Factors associated with the prescription of the antibiotics}

Prescription of meropenem was associated with age, department, length of admission, indication and number of co-morbidities (Table 4). Elderly patients were more likely to be prescribed meropenem than the young ones while having multiple co-morbidities also increased one's likelihood of being prescribed this carbapenem. Similarly, chances of receiving meropenem increased with length of admission as well as having an intra-abdominal, gastrointestinal or central nervous system infection. Ertapenem use was significantly associated with respiratory tract infections $(\mathrm{p}=0.002)$ and being in a medical ward $(\mathrm{p}=<0.001)$ while Piperacillin use was associated with a longer hospital stay $(\mathrm{p}=<0.001)$. An aminoglycoside prescription was associated with younger age $(\mathrm{p}=<0.001)$, admission in the pediatrics wards $(\mathrm{p}=$ $<0.001)$, shorter hospital stays $(\mathrm{p}=0.003)$ as well as having no co morbidities $(\mathrm{p}=<0.001)$.

\section{DISCUSSION}

Among the studied antibiotics, we found Meropenem to be the most commonly prescribed. This is contrary to studies done elsewhere. Vancomycin was found to be the most utilized antibiotic in a study in Romania which corroborated the finding of $\mathrm{S}$. aureus being the prevalent pathogen. ${ }^{6}$ Ciprofloxacin, vancomycin and imipenem were utilized the most in a drug use evaluation in an Iranian hospital, while Imipenem comprised $95 \%$ of all carbapenem prescriptions in yet another study. ${ }^{7,8}$ Present study finding reveals that the restrictive measures put in place by the hospital to limit the use of carbapenems especially meropenem have not succeeded. Among the beta-lactam antibiotics, carbapenems possess the broadest spectrum of activity and are meant to be used as last-line agents or when patients with infections become gravely ill or are suspected of harboring resistant bacteria. ${ }^{9}$ If used empirically for the treatment of patients with serious nosocomial infections of unidentified origin, then this should be for only a limited duration, while awaiting microbiological results. As much as our study found its use to be associated with a prolonged hospital stay, having more than one co-morbidity, as well as admission in critical units, factors that could justify its use, the variance of its use from that of other equally broader spectrum antibiotics is questionably wide. Meropenem is well tolerated and has the advantage of being suitable for administration as an intravenous bolus or infusion. It has also been documented 
to have greater efficacy than ceftazidime or piperacillin/tazobactam in febrile neutropenia, and greater efficacy than ceftazidime plus amikacin or tobramycin in patients with nosocomial pneumonia. ${ }^{10}$ These put it at a selection advantage over the other antibiotics and could explain its increased use in our hospital.

The use of imipenem and ertapenem was quite low in comparison to Meropenem, still signifying a preference for the broader spectrum carbapenem. Imipenem and meropenem are the most established members of the carbapenem class and their current role in therapy remains for use in moderate to severely ill patients with nosocomial and polymicrobial infections. ${ }^{9}$ While imipenem is slightly more active than meropenem against Gram-positive organisms and meropenem slightly more active than imipenem against Gram-negative organisms, direct comparisons between the two drugs report similar bacteriological and clinical cure rates. The only other reported difference is that meropenem is indicated for meningitis while imipenem is not because of its potential to cause seizures. ${ }^{11}$ Therefore, there seems to be a preference for meropenem in our hospital which is most likely due to a larger awareness about it by the prescribers and more experience in its use. It could also be due to a less stringent need to modify doses of Meropenem in situations such as renal impairment. Imipenem requires dose adjustment at creatine clearances below $70 \mathrm{ml} / \mathrm{min}$, compared to meropenem which will not need adjustment until the clearance is below $50 \mathrm{ml} / \mathrm{min}$.

Authors found that aminoglycosides were also predominantly used though their use was more in children. Unpublished reports from our hospital indicate that urinary and respiratory tract infections in this population are due to organisms such as E. coli and Klebsiella which are very sensitive to aminoglycosides. The aminoglycosides were at times prescribed in combination with at least one other antibiotic from another class, especially penicillins and cephalosporins. In these situations, the aminoglycoside was usually added to broaden the spectrum such that gram negative organisms are covered, especially in infections that had failed to respond to the first drug. Amikacin was utilized the most, probably because of a better sensitivity pattern within the hospital (unpublished reports). These findings are consistent with the study by Muller A et al where aminoglycosides were mainly prescribed for the treatment of pulmonary infections, urinary tract infections, and febrile neutropenia and were always prescribed in combination with at least one other antibiotic from another class with a beta-lactam in $94 \%$ of cases. ${ }^{12}$

The duration of antibiotic treatment needs to be individualized taking into account patient variables such as severity of illness, clinical response, and the type of infection. In view of the deleterious effects of prolonged courses of antimicrobial agents, including the potential for adverse reactions, selection of antibiotic-resistant organisms, and high cost, emphasis has been on shorter courses of therapy. ${ }^{2}$ Current guidelines advise a 7-10day course for most infections, unless poor prognosis predictors are present. ${ }^{4,13}$ In other situations, however, a longer duration of therapy is clearly warranted for example 4-6 weeks in intra-abdominal abscesses. At times expert opinion may be sought.

Authors found that cefepime was administered for a mean duration of 6.4 days in the hospital. A similar finding was reported in phase I of a study in Israel before an educational intervention was implemented. ${ }^{14}$ In the present study, Ertapenem was used for a relatively shorter duration than the other broad spectrum antibiotics. It has a half-life of approximately 4hours making it suitable for once-daily administration. Its activity is also more limited primarily because it lacks activity against Pseudomonas aeruginosa and Enterococcus spp. These properties make it more suited to treatment of community-acquired infections and outpatient intravenous antimicrobial therapy than for the treatment of nosocomial infections. ${ }^{9}$ Its use in authors' hospital is most likely for patients with mild to moderate infections who tend to have a shorter hospital stay.

In the present study, meropenem was administered for a mean duration of 6.5 days. This is lower than in two other studies, where the mean duration was 7.3 days and 9.6 days. ${ }^{14,15}$ Considering that meropenem would ideally be used for very severe infections, present study finding may be considered appropriate usage. In fact, use of meropenem was associated with being in a critical care unit. However, there is a growing body of evidence that prolonged antimicrobial therapy is not beneficial for critically ill patients. ${ }^{13}$ The aminoglycosides were used for a mean duration of less than 5 days. This is consistent with the findings from a study in a French university hospital and can be explained by the fact they were used mainly in children with conditions that resolved quickly such as respiratory distress syndrome as well as mild respiratory and urinary tract infections. ${ }^{12}$ Vancomycin had the least duration because it was mainly used for prophylaxis against staphylococcal infections in patients undergoing dialysis.

The commonest indication for the prescription of our study antibiotics were respiratory infections just like the survey in 18 Egyptian hospitals. ${ }^{16}$ Two other studies still found respiratory infections as predominant though at lower prevalence than that in present study. ${ }^{4,14}$ As much as the current study population was restricted to those using the select antibiotics, the finding underpins the fact that respiratory infections are common in Kenya and may account for up to half of all hospital admissions in medical and pediatric wards. ${ }^{17}$

Cultures were done in $62 \%$ of the studied patients. This is lower than in the study in a Norwegian hospital where a microbiological sample was collected prior to 678 out of 939 prescriptions $(72 \%)$ of broad-spectrum antibiotics. ${ }^{18}$ However, 33 patients in present study received these antibiotics for prophylaxis more so following surgical procedures and therefore organism identification wasn't 
necessary in such cases. About a third of patients in two studies had an organism identified. ${ }^{7,19}$ Only 45 out of 187 patients whose samples were taken had a microbiological diagnosis in present study. These low yields can be interpreted in two ways. One is that the patients probably had no bacterial infection to warrant treatment with antibiotics more so those broad spectrum in nature.

The second and more likely reason is that at times samples are drawn for culture and sensitivity when patients have already had one or several courses of antibiotic therapy. Sometimes these antibiotics are initiated within our hospital or the patients come when they have already received them while in another hospital before being referred. Previous antimicrobial therapy decreases the yield and accuracy of cultures for isolation of organisms. ${ }^{4,20}$ In both cases, there is an element of antibiotic misuse which definitely promotes development of resistance. It is therefore important to follow the principles of antimicrobial therapy which dictate that all efforts are made to get a sample for culturing prior to initiation of antibiotics.

Despite the low culture yield in the present study, treatment was de-escalated to a narrow spectrum antibiotic in 22 patients. The remaining patients with a microbiological diagnosis maintained the antibiotics that they had been commenced on provided the organism was sensitive to it, even if for some, the data favored de-escalation. A similar finding was reported in an observational study where cultures were done in $54 \%$ of patients, $23 \%$ de-escalated and $16 \%$ remained unchanged despite results allowing deescalation. $^{21}$

One of the factors limiting this study was its retrospective mode of data collection. Not all information pertaining to patients' treatment was documented. Some complementary information was missing, such as mode of infection acquisition (institutional, home, previous outpatient antibiotic therapy, etc.) as well as full recent antibiotic use history. Equally, authors studied only a few antibiotics. Incorporation of other broad-spectrum antibiotics could probably have led to other findings and explanations that are not mentioned in this article.

\section{CONCLUSION}

To conclude, we emphasize that Meropenem is rampantly used in our hospital more so among adults and those with a prolonged hospital stay. These data highlight the need to strongly intensify implementation of restriction strategies for Meropenem use that may include but not limited to getting authorization from infectious disease specialists before prescribing. Compulsory education programs on antimicrobial stewardship targeting all prescribers and periodic studies on quality of antibiotic prescription should also be considered. We also recommend further studies that will prospectively look at the use of all the broad-spectrum antibiotics, as well as drug susceptibility patterns.

\section{ACKNOWLEDGEMENTS}

Authors would like to thank Dr. Mary Kisingu, Dr Mary Kiarie and the entire pharmacy department of TNH for their support and encouragement during the whole research period. Authors also acknowledge Mr. Nebert Mchidi of TNH School of Nursing and Mr. Conrad Wanyama of Kenya Medical Research Institute for their technical assistance in data analysis and manuscript proof reading.

\section{Funding: No funding sources}

Conflict of interest: None declared

Ethical approval: The study was approved by the Institutional Ethics Committee

\section{REFERENCES}

1. Antimicrobial stewardship: prescribing antibiotics; Guidance and guidelines NICE 2017. Available at: https://www.nice.org.uk/advice/ktt9/chapter/evidence -context.

2. Doron S, Davidson LE. Antimicrobial stewardship. Mayo Clin Proc. 2011;86(11):1113-23.

3. De Waele JJ, Ravyts M, Depuydt P, Blot SI, Decruyenaere J, Vogelaers D. De-escalation after empirical meropenem treatment in the intensive care unit: fiction or reality?. J Critical Care. 2010 Dec 1;25(4):641-6.

4. Leekha S, Terrell CL, Edson RS. General Principles of Antimicrobial Therapy. InMayo Clin Proc. 2011;86(2):156-67.

5. English Surveillance Programme for Antimicrobial Utilisation and Resistance (ESPAUR). 2017;189. Available at: https://assets.publishing.service.gov.uk/government/u ploads/system/uploads/attachment_data/file/656611/ ESPAUR_report_2017.pdf.

6. Bizo PT, Dumitras D, Popa A. Evaluation of restricted antibiotic use in a hospital in Romania. Int $\mathrm{J}$ Clin Pharmacy. 2015 Jun 1;37(3):452-6.

7. Shoaei S, Bagherzadeh A, Haghighi M, Shabani M. Vancomycin and Five Broad-spectrum Antibiotic Utilization Evaluation in an Educational Medical Center in One Year. J Pharmaceuti Care. 2015 Oct 14;2(4):154-61.

8. Jary F, Kaiser JD, Henon T, Leroy J, Patry I, Blasco $\mathrm{G}$, et a. Appropriate use of carbapenems in the Besançon University Hospital. Med Infectious Dis. 2012 Oct 1;42(10):510-6.

9. Papp-Wallace KM, Endimiani A, Taracila MA, Bonomo RA. Carbapenems: past, present, and future. Antimicrob Agents Chemother. 2011;55(11):4943-60.

10. Baldwin CM, Lyseng-Williamson KA, Keam SJ. Meropenem: a review of its use in the treatment of serious bacterial infections. Drugs. 2008;68(6):80338.

11. Zhanel GG, Wiebe R, Dilay L, Thomson K, Rubinstein E, Hoban DJ, et al. Comparative review of the carbapenems. Drugs. 2007;67(7):1027-52. 
12. Muller A, Leroy J, Patry I, Hénon T, Hocquet D, Chirouze C, et al. Appropriateness of aminoglycoside prescriptions in a French university hospital. Medi Infectious Dis. 2016 Sep 1;46(6):308-13.

13. Vincent JL, Bassetti M, François B, Karam G, Chastre J, Torres A, et al. Advances in antibiotic therapy in the critically ill. Critical Care. 2016 Dec;20(1):133.

14. Raveh D, Muallem-Zilcha E, Greenberg A, WienerWell Y, Schlesinger Y, et al. Prospective drug utilization evaluation of three broad-spectrum antimicrobials: cefepime, piperacillin-tazobactam and meropenem. J Assoc Physicians. 2006 May 8;99(6):397-406.

15. Salehifar E, Shiva A, Moshayedi M, Kashi TS, Chabra A. Drug use evaluation of Meropenem at a tertiary care university hospital: a report from Northern Iran. J Res Pharmacy Pract. 2015 Oct;4(4):222-5.

16. Talaat M, Saied T, Kandeel A, El-Ata GA, El-Kholy A, Hafez S, et al. A point prevalence survey of antibiotic use in 18 hospitals in Egypt. Antibiotics. 2014 Sep 10;3(3):450-60.

17. Ndegwa LK, Katz MA, McCormick K, Nganga Z, Mungai A, Emukule G, et al. Surveillance for respiratory health care-associated infections among inpatients in 3 Kenyan hospitals, 2010-2012. Am J Infect Control. 2014 Sep 1;42(9):985-90.

18. Holen $\varnothing$, Alberg T, Blix HS, Smith I, Neteland MI, Eriksen HM. Broad-spectrum antibiotics in
Norwegian hospitals. J Norwegian Med Assoc: Magazine Pract Med, New Series. 2017 Mar;137(5):362-6.

19. van der Velden LBJ, Tromp M, Bleeker-Rovers CP, Hulscher M, Kullberg BJ, Mouton JW, et al. Nonadherence to antimicrobial treatment guidelines results in more broad-spectrum but not more appropriate therapy. Eur J Clin Microbiol Infect Dis. 2012;31(7):1561-8.

20. Kim ES, Kim EC, Lee SM, Yang SC, Yoo CG, Kim $\mathrm{YW}$, et al. Bacterial yield from quantitative cultures of bronchoalveolar lavage fluid in patients with pneumonia on antimicrobial therapy. Korean J Internal Med. 2012 Jun;27(2):156.

21. Álvarez-Lerma F, Alvarez B, Luque P, Ruiz F, Dominguez-Roldan JM, Quintana E, et al. Empiric broad-spectrum antibiotic therapy of nosocomial pneumonia in the intensive care unit: a prospective observational study. Critical Care. 2006 Jun;10(3):R78.

Cite this article as: Mariita KM, Chirima HA, Maina CK. Broad spectrum antibiotic use among inpatients at a hospital in Nairobi, Kenya. Int J Basic Clin Pharmacol 2019;8:1-7. 